\title{
Acute schistosomiasis diagnosis: a new tool for the diagnosis of schistosomiasis in a group of travelers recently infected in a new focus of Schistosoma mansoni
}

\author{
Rafaella Fortini Queiroz Grenfell[ ${ }^{[1]}$, Watson Martins ${ }^{[1]}$, Sandra Costa Drummond ${ }^{[2]}$, \\ Carlos Maurício de Figueiredo Antunes ${ }^{[3]}$, Izabela Voieta ${ }^{[4]}$, Alba Otoni ${ }^{[4]}$, \\ Áureo Almeida de Oliveira ${ }^{[1]}$, Vanessa Silva-Moraes ${ }^{[1]}$, Eduardo Ribeiro de Oliveira ${ }^{[1]}$, \\ Edward Oliveira ${ }^{[5]}$, José Roberto Lambertucci ${ }^{[4]}$, Cristina Toscano Fonseca ${ }^{[1]}$ \\ and Paulo Marcos Zech Coelho[1]
}

[1]. Laboratório de Esquistossomose, Centro de Pesquisas René Rachou, Fundação Oswaldo Cruz, Belo Horizonte, MG. [2]. Fundação Nacional de Saúde, Belo Horizonte, MG. [3]. Santa Casa de Misericórdia de Belo Horizonte, Belo Horizonte, MG. [4]. Serviço de Doenças Infecciosas e Parasitárias, Faculdade de Medicina, Universidade Federal de Minas Gerais, Belo Horizonte, MG. [5]. Laboratório de Pesquisas Clínicas, Centro de Pesquisas René Rachou, Fundação Oswaldo Cruz, Belo Horizonte, MG.

\begin{abstract}
Introduction: The diagnosis of schistosomiasis mansoni on early stages of infection is important to prevent late morbidity. A simple, cheap, sensitive and specific assay for routine diagnosis of schistosome infection based on the detection of specific IgG for schistosomula tegument antigens (ELISA-SmTeg) was developed by our group. Methods: We describe here an acute outbreak involving a travel group of 80 individuals from a non-endemic area of the State of Minas Gerais, Brazil. These individuals were in contact with a freshwater pool where Biomphalaria glabrata was found. Results obtained from our new methodology were compared to IgG antibody titers against soluble worm antigenic preparation (SWAP) by ELISA and, also to parasitological examination, nuclear magnetic resonance and clinical findings. Results: ELISA-SmTeg was capable of detecting 64 positive cases among the 80 individuals participating at the survey with a positivity ratio of $80 \%$ and a higher sensitivity than ELISA-SWAP that was only sensitive for $56 \%$ of positive cases. Besides, a significant correlation was found for the severity of the infection and the specific IgG titers against SmTeg. Conclusions: Our data showed that ELISA-SmTeg might serve as the initial diagnostic tool for acute stages of the infection in community-based helminth control programs or for the surveillance of individuals from non-endemic areas.
\end{abstract}

Keywords: Schistosomiasis mansoni. Immunological diagnosis. Schistosomula antigens. Acute outbreak.

\section{INTRODUCTION}

Schistosomiasis continues to be a major worldwide public health problem that affects 200 million people and about 779 million people live in endemic areas in the Middle East, South America, Caribbean, Southeast Asia and particularly subSaharan Africa ${ }^{1}$. Nonetheless, the disease is seen in growing numbers in recently detected foci of transmission due to increased immigration from endemic areas and tourism. Severe sequelae due to schistosomiasis are rare in travelers from non-endemic areas because the risk of developing significant pathological manifestations after short-term exposure that frequently result in high parasite burden is limited by the life span of the adult worm. The most important factor regarding

Address to: Dr. Paulo Marcos Zech Coelho. Lab. Esquistossomose/CPqRR/ FIOCRUZ. Av. Augusto de Lima 1715, 30190-002 Belo Horizonte, MG, Brasil.

Phone: 5531 3349-7740; Fax: 5531 3349-7700

e-mail: coelhopm@cpqrr.fiocruz.br

Received 29 November 2012

Accepted 26 March 2013 the difficulty of controlling the spread of the disease is the absence of diagnostic methods capable of detecting the disease, especially in the early patent phase.

Clinical findings make possible the division of the disease in two phases. First is the initial phase, which includes cercarian dermatitis caused by the penetration of cercariae into the skin and the local presence of schistosomula. Together, the acute symptomatic schistosomiasis that may appear 3 to 7 weeks after an exposure and is characterized by cough, fever, anorexia, abdominal pain and headaches. In this phase, the inflammatory reaction is well established and involves predominantly Th1 bias, when immunoglobulin levels are elevated in the serum ${ }^{2}$ and hypereosinophilia. The late acute phase that occurs after 40 to 60 days post-infection is the acute phase, characterized by the egg laying and a predominantly $\mathrm{Th} 2$ inflammatory response. Its clinical manifestations vary depending on the parasite load and host immune response. Patients may present hepatointestinal, hepatosplenic, schistosomal myeloradiculopathy and acute forms with the latter being a main indicator of schistosomiasis severity ${ }^{3,4}$.

Although treatment with oxamniquine and artemisinin on the first days after infection is considered more efficient in obtaining cure than praziquantel ${ }^{5-8}$, the lack of methods to detect 
the initial phase, before egg laying, precludes the patients from a successful therapy. The diagnosis is first guided by the patient history of water contact in an endemic area. This evidence is further confirmed by the presence of the Schistosoma mansoni eggs in stool samples after egg laying, on oviposition phase ${ }^{9}$. Even during early patent phase, the absence of $S$. mansoni eggs in the faeces does not rule out the diagnosis due to the low sensitivity this methodology may present. There is usually a miliary distribution of eggs in the organs of the host and laparoscopy frequently reveals whitish nodules, as granulomas, on the surface of liver, intestines and visceral peritoneus. Hence, it is expected that the parasitological methods do not have properly efficiency for the diagnosis, predominantly for patients in acute phase once the female has not yet laid eggs ${ }^{2}$.

Serological techniques have proven advantages in diagnosis of schistosomiasis ${ }^{10-12}$ since the humoral response especially related to IgG antibodies in acute patients to egg and worm antigens does not differ from the chronic phase as a significant levels of antibodies are detected, but they are not yet part of the routine of control programs ${ }^{13}$.

This work focus on the evaluation of an indirect enzyme linked immunosorbent assay using schistosomula tegument antigen (ELISA-SmTeg). This methodology was first standardized using 7 to 10 days post-infection mice sera when it showed to be a promising diagnostic tool for the acute phase of the disease. In this report, we describe a case involving a group of travelers in Brazil that was exposed to a contaminated freshwater pool. Early detection of the clinical symptoms or signs of schistosomiasis mansoni combined with rapid investigation of the entire group enabled us to observe this group prospectively. The main goal of this work is to offer a new reliable diagnostic tool to determine positive cases before egg laying.

\section{METHODS}

\section{Study population}

Eighty individuals were part of a group that was hosted on a country house in Colônia do Teodoro, a recently endemic focus for schistosomiasis mansoni, next to the City of São João Del Rei in the State of Minas Gerais, in southeast Brazil, from December/2009 to February/2010. The individuals were exposed to a freshwater pool where Biomphalaria glabrata snails were found.

\section{Identifying cases}

To identify symptomatic cases, we initiated the interviews with exposed travelers on March/2010 when samples were collected (1 to 3 months post-infection). Follow-up focused on the detection of symptoms or signs of chronic schistosomiasis previously described in travelers, together with current symptoms and signs, including pulmonary symptoms, gastrointestinal symptoms (e.g. abdominal pain or diarrhea), constitutional symptoms (e.g. fatigue or weight loss), and myeloradiculopathy involvement.
Schistosoma mansoni infection was defined as exposure to the freshwater pool plus one of the following criteria: presence of eggs in faecal samples, IgG antibody titers by ELISA using adult worm antigens, myeloradiculopathy detected by nuclear magnetic resonance, and/or symptoms compatible with acute schistosomiasis. Symptoms defining infection in this group were fever, cough, cercarial dermatitis, and angioedema. Nonspecific symptoms (e.g. fatigue, gastrointestinal complaints, and headache) were reported as well. Blood samples were collected by venipuncture of all those patients. Individual serum samples were obtained after centrifugation of blood samples at $3,000 \mathrm{~g}$ for $5 \mathrm{~min}$. These samples were maintained at $-20^{\circ} \mathrm{C}$. Twenty four out of 80 individuals agreed to submit faeces for examination and these samples were firstly analyzed by Kato-Katz parasitological assay ${ }^{14}$. Three glass slides $(41.7 \mathrm{mg} /$ smear) of a single fecal sample were examined. Five patients were hospitalized, one with advanced pulmonary stage, one with schistosomal myeloradiculopathy and, three with severe dehydration caused by the hepatointestinal form.

Positive individuals for one of the described criteria were treated with a single oral dose of praziquantel $(50 \mathrm{mg} / \mathrm{kg})$, according to the recommendation of the Brazilian Ministry of Health.

\section{ELISA-SWAP}

Enzyme-linked immunosorbant assay with adult soluble worm antigenic preparation (ELISA-SWAP) was performed in microtiter plates MaxiSorp Surface (NUNC, Denmark) sensitized with $100 \mu \mathrm{l} / \mathrm{well}$ of $1 \mu \mathrm{g} / \mathrm{ml}$ of SWAP diluted in buffer $0.05 \mathrm{M}$ carbonate-bicarbonate $\mathrm{pH}$ 9.6. The incubation was done for $16 \mathrm{~h}$ at $4^{\circ} \mathrm{C}$. The plates were washed three times with $0.15 \mathrm{M}$ phosphate buffer saline $\mathrm{pH} 7.2$ with $0.05 \%$ of Tween 20 (LGC Biotecnologia, BR) (washing buffer) and, the non-specific sites were blocked with $10 \%$ fetal bovine serum in washing buffer at $37^{\circ} \mathrm{C}$ for $1 \mathrm{~h}$. After new washing steps, $100 \mu$ l of human serum samples diluted 1:50 in PBS were added in triplicate into each well and the plates were incubated at room temperature for $1 \mathrm{~h}$. Following, the plates were submitted to washing steps and incubated at room temperature for $1 \mathrm{~h}$ with conjugated anti-IgG human peroxidase (Southern Biotech, USA) diluted 1:60,000 in washing buffer. The plates were washed again and $100 \mu$ l of substrate 3,3',5,5-tetramethylbenzidine solution (TMB/H2O2) (Invitrogen, USA) were added to each well. The reaction was stopped after $20 \mathrm{~min}$ of incubation in the dark by addition of $50 \mu \mathrm{l} /$ well of $2 \mathrm{~N}$ sulfuric acid. The results were obtained as absorbance values at $450 \mathrm{~nm}$ in microplate reader (Bio-Rad Laboratories 3550, JA). The cut off value of the ELISA-SWAP was $\mathrm{OD}=0.188$ (standard deviation of 0.08$)$, determined by ROC curve $(A=0.70, p<0.0001)$.

\section{Groups of study}

The 80 individuals were divided in four groups according to diagnosis results. Group 1 were limited to patients with symptoms and/or signs of acute schistosomiasis but negative for any other diagnostic method used (total of 28 individuals) whereas, group 2 , with 26 individuals, involved patients that were positive for clinical examination plus ELISA-SWAP. Patients with positive results for these two last criteria that additionally presented 
in stools constituted group 3, with a total of 21 individuals. Finally, all the five hospitalized patients with severe schistosomiasis were represented by group 4 .

\section{Evaluation of the indirect enzyme-linked immunosorbent assay using schistosomula tegument antigen (ELISA-SmTeg)}

Serologic studies were conducted at the Brazilian Excellence Center for the Diagnosis of Schistosomiasis mansoni, Oswaldo Cruz Foundation (Brazilian Ministry of Health) in Belo Horizonte. The serologic test performed is based on the detection of specific immunoglobulin $\mathrm{G}(\mathrm{IgG})$ against schistosomula tegument antigen by ELISA methodology.

Preparation of Schistosoma mansoni schistosomula tegument antigen: cercariae of the LE strain were obtained at the Laboratory of Malacology of the René Rachou Research Center, Oswaldo Cruz Foundation and were mechanically transformed into schistosomula ${ }^{15}$. Briefly, cercariae were placed into conical tubes and left in ice bath for $30 \mathrm{~min}$ before centrifugation (Eppendorf Centrifuge 5820R, Hamburg, GR) at $200 \mathrm{G}$ for $3 \mathrm{~min}$ at $4^{\circ} \mathrm{C}$. The pellet was ressuspended in cold Earl's salts plus lactalbumin hydrolyzate medium. The cercarial tails were broken in vortex (Scientific Industries Genie-2, New York, USA) for $2 \mathrm{~min}$. Later the tails were removed from the medium through repeated washing steps with Earl's salts media at $37^{\circ} \mathrm{C}$, and schistosomula were incubated for $90 \mathrm{~min}$ at $37^{\circ} \mathrm{C}$. This step was followed by centrifugation at $200 \mathrm{G}$ for $1 \mathrm{~min}$. For tegument removal, $2 \mathrm{ml}$ of $0.3 \mathrm{M}$ calcium chloride were added to the schistosomula that was stirred in vortex for $7 \mathrm{~min}$ and centrifuged at $200 \mathrm{G}$ for $1 \mathrm{~min}$. The supernatant was centrifuged at 50,000 $\mathrm{G}$ for an hour and the pellet enriched of membrane was ressuspended in $0.9 \%$ saline and dialyzed against $1.7 \%$ saline solution for $72 \mathrm{~h}$. Protein concentration was determined by Bradford method ${ }^{16}$. The final concentration used in standardized tests was $0.52 \mathrm{mg} / \mathrm{ml}$.

Indirect enzyme-linked immunosorbent assay: microtiter plates MaxiSorp surface were coated with $100 \mu \mathrm{l}$ per well of SmTeg $(1 \mu \mathrm{g} / \mathrm{ml})$ in buffer $0.05 \mathrm{M}$ carbonate-bicarbonate $\mathrm{pH} 9.6$ for $16 \mathrm{~h}$ at $4^{\circ} \mathrm{C}$. After plates were washed, the free sites were saturated with $300 \mu \mathrm{l}$ per well of $2.5 \%$ skim milk diluted in washing buffer, incubating at $37^{\circ} \mathrm{C}$ for $1 \mathrm{~h}$ and $100 \mu$ l of individual serum sample (diluted $1: 100$ ) in $0.15 \mathrm{M}$ phosphate buffer saline $\mathrm{pH} 7.2$ were added to the plates, followed by incubation for $1 \mathrm{~h}$. The plates were submitted to washing steps and incubated for $1 \mathrm{~h}$ with anti-IgG conjugated to peroxidase diluted in washing buffer (anti-human IgG Fc specific peroxidase) at the dilution of 1:60,000. Then, $100 \mu$ of substrate solution were added to each well and the enzymatic reaction was stopped after 10min by adding $50 \mu \mathrm{l}$ per well of $2 \mathrm{~N}$ sulfuric acid. Results were obtained as absorbance values at $450 \mathrm{~nm}$. The cut off value of the ELISA-SmTeg was $\mathrm{OD}=0.110$ (standard deviation of 0.02 ), determined by ROC curve $(\mathrm{A}=0.92, \mathrm{p}<0.0001)$.

Negative controls were properly assayed using fifty three volunteers' serum samples as our control group, also wells without antigen and serum samples as control of nonspecific adsorption of conjugate. The standard dilution of sera and conjugated antibodies was determined by a dilution curve, performed with the same reagents and equipment based on six different dilutions.

Statistical analysis: data deriving from absorbance values were analyzed with Minitab software (Minitab Inc, College, USA) by Kolmogorov-Smirnov normality test. Normal distributed data were analyzed by Student's $t$ test and comparisons between methods were done by Chi-square analysis $(\mathrm{p}<0.05$ as significance level). The sensitivity, specificity, cut off values, receiver operating characteristic (ROC) curve and positivity ratios, defined as the capability of detecting real positive and negative individuals, were determined with Prism 5.0 software.

\section{Ethical considerations}

The Ethical Committee of Fundação Nacional de Saúde in Minas Gerais, Brazil granted ethical approval for this study. Written informed consent was obtained from each individual.

\section{RESULTS}

All the 80 individuals hosted on the country house that had contact with the freshwater pool participated on this survey. Serum samples were submitted to a well established indirect ELISA-SWAP assay that allowed us to identify the levels of specific IgG of each individual patient and a correlation was done with the new assay ELISA-SmTeg, which has been previously standardized in our laboratory for the determination of specific IgG antibody levels against schistosomula tegument antigen.

The cut off value for this new assay had been previously determined $(\mathrm{OD}=0.110)$ using fifty three volunteers' serum samples as our control group by the ROC curve $(A=0.92$, $\mathrm{p}<0.0001$ ), as shown on Figure 1. It is important to notice that the high sensitivity described was determined with clinical acute phase patients and the same effectiveness was not seen with the chronic form patients (data not shown).

Using the defined cut off value, samples from the 80 individuals were applied on ELISA-SmTeg assay and a first comparison was done with data obtained from another indirect immunoassay usually used as the routine method, the ELISASWAP. Table 1 shows the comparison between results obtained from both ELISA assays. Among the 80 positive patients, only 45 individuals were properly diagnosed by ELISA-SWAP. On the other hand, ELISA-SmTeg showed a higher sensitivity by being capable of identifying 64 positive individuals $(\mathrm{p}=0.001)$. The positivity ratio of ELISA-SmTeg assay of $80 \%$ was superior to $56 \%$ obtained by ELISA-SWAP. The Cohen's Kappa Index of 0.385 (standard deviation of \pm 0.094 ) indicates fair agreement between both immunological assays.

Fecal samples were obtained in 24/80 (30\%) persons, and eggs were detected in $24 / 24(100 \%)$ fecal samples by Kato-Katz. The patients, additionally, demonstrated high levels of specific IgG antibodies for ELISA-SmTeg with an average of 0.401 (standard deviation of \pm 0.016$)$. Three $(13 \%)$ positive patients 


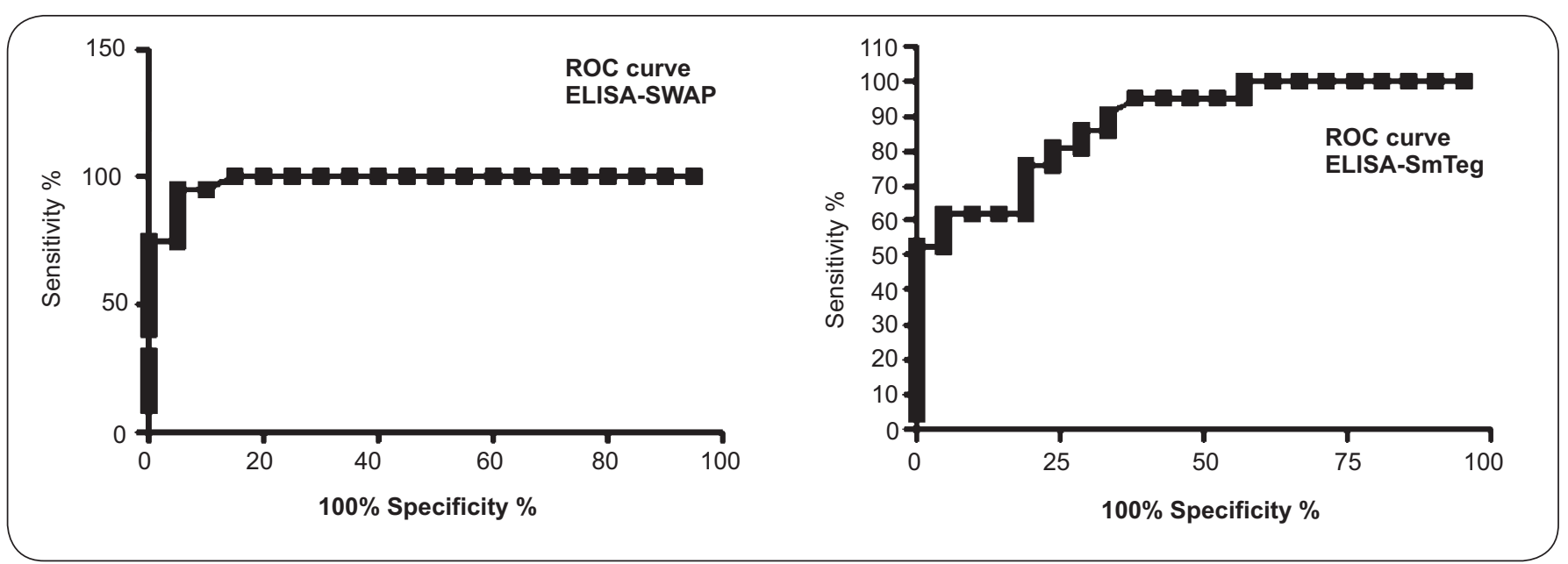

FIGURE 1 - Receiver operating characteristic curve of ELISA-SWAP and ELISA-SmTeg assays determined with sera samples from health volunteers and infected patients. Cut off values are $\mathrm{OD}=0.188$ and $\mathrm{OD}=0.110$, respectively for ELISA-SWAP and ELISA-SmTeg. Artwork created by Prism 5.0 software. ROC: receiver operating characteristic; ELISA-SWAP: enzyme-linked immunosorbant assay-soluble worm antigenic preparation; ELISA-SmTeg: ELISA-schistosomula tegument antigens; OD: optical density.

with eggs in stools were not detected by ELISA-SWAP showing an OD average of 0.157 ( \pm 0.026$)$.

Patients with clinical symptoms and/or signs that were properly diagnosed by both methods, Kato-Katz and ELISASWAP (21/80) (26\%), showed also high levels of IgG against SmTeg $(21 / 21)(100 \%)$. The IgG levels for these patients $(26 / 80)$ were significantly higher (33\%) than from patients whom were positive only for ELISA-SWAP, presenting no eggs in stools $(\mathrm{p}=0.002)$. Figure 2 shows this relation after patients were divided in four individual groups. We could see that patients that was diagnosed as negative by ELISA-SWAP, but presented all the clinical symptoms compatible with acute schistosomiasis (28/80) (35\%), also presented lower IgG titers for SmTeg in comparison to group $2(\mathrm{p}=0.002)$. The analysis of the patients with severe schistosomiasis (group 4), one with advanced pulmonary stage, one with schistosomal myeloradiculopathy and, three with severe intestinal form, allows us to reaffirm that ELISA-SmTeg presented once more a high positivity ratio by being capable of diagnosing all the severe cases $(5 / 80)(6 \%)$ and, additionally presented a correlation between specific IgG levels and the severity of the infection. Statistical differences were found when comparisons were made for the antibody level of patients on groups 1 and 2 in comparison to group 4 ( $\mathrm{p}=0.003$ and 0.001 , respectively).

TABLE 1 - Diagnosis results of enzyme-linked immunosorbant assay with adult worm antigens and with schistosomula tegument antigen of serum samples from the 80 travelers positive for schistosomiasis mansoni

\begin{tabular}{lcc}
\hline & \multicolumn{2}{c}{ Diagnosis of travelers } \\
\cline { 2 - 3 } & ELISA-SWAP & ELISA-SmTeg \\
\hline Positive & 45 & $64^{* * *}$ \\
Negative & 35 & 16 \\
\hline Total & 80 & 80 \\
\hline
\end{tabular}

ELISA-SWAP: ELISA-soluble worm antigenic preparation; ELISA-SmTeg: ELISAschistosomula tegument antigens. Statistical differences between the number of positive individuals by both assays are represented by $(* * *)(\mathrm{p}=0.001)$ after Chi-square analysis. Artwork created by Prism 5.0 software.

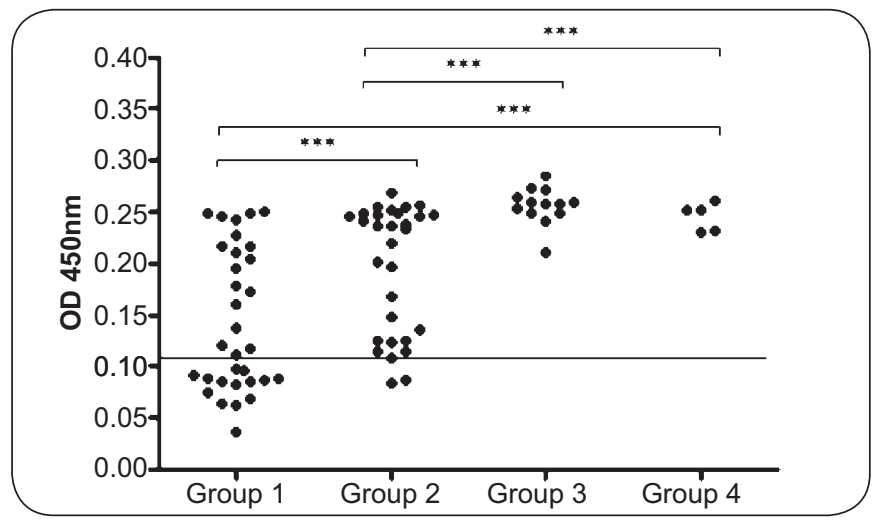

FIGURE 2 - Relation between the number of travelers diagnosed by ELISASmTeg and groups divided according to the additional diagnostic results and severity of infection. Group 1: 28 patients positive only for clinical examination; Group 2: 26 patients positive for clinical examination plus ELISA-SWAP; Group 3: 21 patients positive for clinical examination, ELISA-SWAP and Kato-Katz; Group 4: 5 hospitalized patients with severe schistosomiasis. Line represents the cut off of $\mathrm{OD}=0.110$ for ELISA-SmTeg. Results represent the mean of four absorbance values at $450 \mathrm{~nm}$. Statistical differences between groups are represented by $(* * *)$ after Student $\mathrm{t}$ test, for $\mathrm{p}=0.002$ and 0.003 , respectively for groups 1 and 2 and, groups 1 and 4 ; $\mathrm{p}=0.002$ and 0.001 , for groups 2 and 3 and, groups 2 and 4 . Artwork created by Prism 5.0 software. ELISA-SmTeg: enzyme-linked immunosorbant assay-schistosomula tegument antigens; ELISA-SWAP: enzyme-linked immunosorbant assay-soluble worm antigenic preparation; OD: optical density.

\section{DISCUSSION}

Acute schistosomiasis is one of the clinical manifestations of infection with Schistosoma sp. ${ }^{17}$. The pre-postural phase occurs between cercarial penetration in skin and egg laying by female adult worms after the migration of young parasite to portal system and the maturation of male and female worms ( 7 to 8 weeks). Oviposition, which defines the postural phase of the disease, typically occurs 45 to 60 days after cercariae penetration. This symptomatic acute phase is mostly severe in non-endemic (primary infection) individuals (i.e. tourists, travelers) exposed to fresh water in endemic areas ${ }^{17-19}$. 
It is considered to be a toxemic and allergic reaction to the migrating and maturing schistosomula ${ }^{20}$. The severity of the clinical presentation varies according to the worm burden and the immune response to the released parasite antigens ${ }^{6}$.

Circulating immune complexes are found in 55-93\% of patients with acute schistosomiasis, and their presence and amount are correlated to the intensity and severity of symptoms ${ }^{21,22}$. These symptoms are typically seen before oviposition, and the appearance of granulomatous reactions around eggs. Oviposition begins at the end of adult maturation and migration to the vesical plexus or mesenteric veins ${ }^{23}$. In the early stages of the acute phase (until 40 days of infection), a search for eggs in stools will be negative. Nonetheless, eggs may still be detected in stools of patients complaining of symptoms compatible with clinical acute form (45-50 days post-infection), although the Kato-Katz technique is rather insensitive in light infections ${ }^{18}$.

Serological testing is an important diagnosis tool to be consider in acute schistosomiasis, especially for the diagnosis before egg laying. Still, it is crucial to select an antigenic portion of the helminth in order to solve the problem of variability on the sensitivity that may occur according to the antigen used. Even so, the primary investigation must be done by a physician in order to determine the medical history of each individual case and then, serological findings ought to be used as a confirmatory methodology. Among the immunological assays available, ELISA is the most commonly used, especially with egg or worm antigens. Its methodology sensitivity can be high for patients with chronic form, reaching values of $89-96 \%$ for egg antigens and $90-94 \%$ for worm antigens, which is related to the cut off titre defining positivity ${ }^{24,25}$. It is also possible to use a combination of serological testes and/or antigens, such as SEA and SWAP, to simultaneously establish the diagnosis of schistosomiasis and discriminate the acute from the chronic forms of the disease and to increase sensitivity ${ }^{26}$. Nonetheless, early treatment with praziquantel, although partially active for immature schistosome stages, should be ready for exposed travelers after diagnosis ${ }^{6,7,27}$.

Reports of outbreaks in travelers with a single and recent exposure have contributed to a better description of the natural history of schistosomiasis and, particularly in those cases, diagnosis relies mainly on positive serological testing. We report here a recent focus of schistosomiasis mansoni involving 80 individuals that had contact with a contaminated freshwater pool. As a consequence, all the individuals presented clinical findings compatible with acute schistosomiasis and were further investigated for IgG antibody levels by ELISA-SWAP. Although the Kato-Katz analysis was not performed for all the 80 patients, the sensitivity of ELISA-SWAP was low when it is being used as a tool for the immunodiagnosis of patients with acute form.

Thus, based on the statement that difficulties associated with clinical or parasitological diagnosis in early patent phase might be overcome by adoption of immunological methods ${ }^{28}$, we developed a new indirect ELISA methodology using schistosomula antigen based on the helminth life-cycle. Data from ELISA-SmTeg showed a significant positivity ratio when 64/80 patients presented high levels of specific IgG, superior to ELISA-SWAP results (45/80), that is the most used immunological method. Positivity ratio was $56 \%$ and $80 \%$, respectively for ELISA-SWAP and ELISA-SmTeg. This final analysis corroborates with our first findings showing that ELISA-SmTeg is more sensitive for the differential diagnosis of patients with acute schistosomiasis. There is generally a trade-off between sensitivity and specificity, and the relative performance of a new diagnostic test is therefore calculated as an index in which both these parameters are accounted for. Sensitivity, however, has more relevance in a public health context, especially in situations where the goal is the complete elimination of a new focus ${ }^{28}$.

When comparing the Kato-Katz parasitological result of each individual patient to the specific IgG antibody level for ELISA-SmTeg, once again, the effectiveness of our new immunoassay was confirmed when the assay had properly diagnosed all these 24 positive patients. On the other hand, ELISA-SWAP did not achieve the same effectiveness after 3 patients with eggs in stools were not differently diagnosed showing low IgG titers.

When patients were divided into groups based on the results obtained by different diagnostic methodologies and on the severity of infection, it could be seen that the $\operatorname{IgG}$ titers against $\mathrm{SmTeg}$ antigen significantly increase as the positivity ratio became higher. Individuals that were positive only for the clinical examination (group 1) showed significant lower levels of IgG antibody than those that were also positive for ELISASWAP assay (group 2). Accordingly, patients that were positive for Kato-Katz other than clinical examination and ELISA-SWAP (group 3) demonstrated significant higher IgG titers in sera than group 1 and group 2. No doubt that including clinical findings as a key to look closely for evidence of infection is important in patient management, as eosinophil count. But correlating immunodiagnostic results and antibody levels with clinical data should allow us to improve our practicing in rural endemic areas, which in turn would significantly enhance the efficiency of the anti-schistosomiasis control program ${ }^{29}$.

Finally, five hospitalized patients with the severe form of the infection were positive for the presence of eggs in stool and, once more, presented high levels of specific IgG determined by ELISA-SmTeg corroborating with the initial findings that shows a significant sensitivity for this new immunoassay. A positivity ratio of $80 \%$ was observed for ELISA-SmTeg and a correlation was confirmed when comparing IgG levels against SmTeg and the severity of the infection. Furthermore, the low sensitivity for ELISA-SWAP was again reported when the assay missed the diagnosis of two patients with the severe form of the infection.

While antibody-based methods suffer from low sensitivity, especially for the diagnosis of acute schistosomiasis ${ }^{2}$, the ELISA-SmTeg was noticed here as an important tool to confirm real recently infected individuals during the acute phase. This new tool was superior than the usual ELISA-SWAP assay, being also able to be used as a tool for measuring the severity of the infection. This new diagnostic method should be especially considered for groups from non-endemic areas, 
such as tourism and social/official activities, including military visits on endemic areas. Hence, an immunoassay capable of diagnosing individuals with acute form, especially before the egg laying will allow the increase on the control programme's effectiveness ${ }^{28}$. Since ELISA-Smteg and ELISA-SWAP do no correlate perfectly, these antigens could be used in parallel to further increase sensitivity in a given population.

\section{CONFLICT OF INTEREST}

The authors declare that there is no conflict of interest.

\section{FINANCIAL SUPPORT}

Conselho Nacional de Desenvolvimento Cientifico e Tecnológico (CNPq), Fundação de Amparo à Pesquisa do Estado de Minas Gerais (FAPEMIG), Fundação Oswaldo Cruz (FIOCRUZ)/Centro de Pesquisas René Rachou (CPqRR), Coordenação de Aperfeiçoamento de Pessoal de Nivel Superior (CAPES) and The Council of the International Educational Exchange of Scholars (Fulbright, U.S. Department of State). The funders had no role in study design, data collection and analysis, decision to publish, or preparation of the manuscript.

\section{REFERENCES}

1. World Health Organization. The social context of schistosomiasis and its control: an introduction and annotated bibliography; 2008. [Cited 2012 April 10]. Available from: http://dosei.who.int/uhtbin/cgisirsi/tLEqou8myB/26990009/5/0.

2. Lambertucci J. Acute schistosomiasis mansoni: revisited and reconsidered. Mem Inst Oswaldo Cruz 2010; 105:422-435.

3. Lambertucci JR, Serufo JC, Gerspacher-Lara R, Rayes AA, Teixeira R, Nobre V, et al. Schistosoma mansoni: assessment of morbidity before and after control. Acta Trop 2000; 77:101-109.

4. Pereira LF, Gazzaneo AL, Melo RM, Tenório HC, Oliveira DS, Alves MS, et al. Clinical and laboratory evaluation of schistosomiasis mansoni patients in Brazilian endemic areas. Mem Inst Oswaldo Cruz 2010; 105:449-453.

5. García Goyco C, Nazario-López H, Ruiz-Tiben E, Vázquez G, Borges T, Nash TE. The efficacy of oxamniquine in acute schistosomiasis: a clinical analysis of 28 treated patients. Bol Asoc Med PR 1997; 89:63-69.

6. Coelho PM, Enk MJ, Katz N. Treatment of clinical schistosomiasis at the prepatent phase: an option? Trends Parasitol 2009; 25:299-300.

7. Enk MJ, Katz N, Coelho PMZ. A case of Schistosoma mansoni infection treated during the prepatent period. Nature Clin Pract Gastroenterol \& Hepatol 2008; 5:112-115.

8. Liu R, Dong HF, Guo Y, Zhao QP, Jiang MS. Efficacy of praziquantel and artemisinin derivatives for the treatment and prevention of human schistosomiasis: a systematic review and meta-analysis. Parasit Vectors 2011; 4:201.

9. Berhe N, Medhin G, Erko B, Smith T, Gedamu S, Bereded D, et al. Variations in helminth faecal egg counts in Kato-Katz thick smears and their implications in assessing infection status with Schistosoma mansoni. Acta Trop 2004; 92:205-212.
10. Turner P, Lalloo K, Bligh J, Armstrong M, Whitty CJ, Doenhoff MJ, et al. Serological speciation of human schistosome infections by ELISA with a panel of three antigens. J Clin Pathol 2004; 57:1193-1196.

11. Gonçalves MM, Barreto MG, Peralta RH, Gargioni C, Gonçalves T, Igreja RP, et al. Immunoassays as an auxiliary tool for the serodiagnosis of Schistosoma mansoni infection in individuals with low intensity of egg elimination. Acta Trop 2006; 100:24-30.

12. Oliveira EJ, Kanamura HY, Takei K, Hirata RD, Valli LC, Nguyen NY, et al. Synthetic peptides as an antigenic base in an ELISA for laboratory diagnosis of schistosomiasis mansoni. Trans R Soc Trop Med Hyg 2008; 102:360-366.

13. Caldas IR, Campi-Azevedo AC, Oliveira LF, Silveira AM, Oliveira RC, Gazzinelli G. Human schistosomiasis mansoni: immune responses during acute and chronic phases of the infection. Acta Trop 2008; 108:109-117.

14. Katz N, Chaves A, Pellegrino J. A simple device for quantitative stool thicksmear technique in schistosomiasis mansoni. Rev Inst Med Trop Sao Paulo 1972; 14:397-400.

15. Ramalho-Pinto FJ, Gazzinelli G, Howells RE, Mota-Santos TA, Figueiredo EA, Pellegrino J. Schistosoma mansoni: defined system for stepwise transformation of cercariae to schistossomule in vitro. Exp Parasitol 1974; 36:360-372.

16. Bradford MM. A rapid and sensitive method for the quantitation of microgram quantities of protein utilizing the principle of protein-dye binding. Anal Biochem $1976 ; 7: 248-254$.

17. Ross AG, Vickers D, Olds GR, Shah SM, McManus DP. Katayama syndrome. Lancet Infect Dis 2007; 7:218-224.

18. Jauréguiberry S, Paris L, Caumes E. Acute schistosomiasis, a diagnostic and therapeutic challenge. Clin Microbiol Infect 2010; 16:225-231.

19. Gryseels B, Polman K, Clerinx J, Kestens L. Human schistosomiasis. Lancet 2006; 368:1106-1118.

20. Corachan M. Schistosomiasis and international travel. Clin Infect Dis 2002; 35:446-450.

21. Hiatt RA, Sotomayor ZR, Sanchez G, Zambrana M, Knight WB. Factors in the pathogenesis of acute schistosomiasis mansoni. J Infect Dis 1979; 139:659-666.

22. Hiatt RA, Ottesen EA, Sotomayor ZR, Lawley TJ. Serial observations of circulating immune complexes in patients with acute schistosomiasis. J Infect Dis 1980; 142:665-670.

23. Southgate VR, Bray RA. Medical helminthology. In: Cook GC, Zumla A, editors. Manson's tropical diseases. 22 ${ }^{\text {nd }}$ ed. London: Saunders Elsevier; 2003.

24. Van Gool T, Vetter H, Vervoort T, Doenhoff MJ, Wetsteyn J, Overbosch D. Serodiagnosis of imported schistosomiasis by a combination of a commercial indirect hemagglutination test with Schistosoma mansoni adult worm antigens and an enzyme-linked immunosorbent assay with S. mansoni egg antigens. J Clin Microbiol 2002; 40:3432-3437.

25. Sorgho H, Bahgat M, Poda JN, Song W, Kirsten C, Doenhoff MJ, et al. Serodiagnosis of Schistosoma mansoni infections in an endemic area of Burkina Faso: performance of several immunological tests with different parasite antigens. Acta Trop 2005; 93:169-180.

26. Beck L, Van-Lüme DS, Souza JR, Domingues AL, Favre T, Abath FG, et al. Discriminating acute from chronic human schistosomiasis mansoni. Acta Trop 2008; 108:229-233.

27. Enk MJ, Lima AC, Drummond SC, Schall VT, Coelho PM. The effect of the number of stool samples on the observed prevalence and the infection intensity with Schistosoma mansoni among a population in an area of low transmission. Acta Trop 2008; 108:222-228.

28. Doenhoff MJ, Chiodini PL, Hamilton JV. Specific and sensitive diagnosis of schistosome infection: can it be done with antibodies? TRENDS in Parasitology 2004; 20:35-39.

29. Al-Sherbiny MM, Osman AM, Hancock K, Deelder AM, Tsang VC. Application of immunodiagnostic assays: detection of antibodies and circulating antigens in human schistosomiasis and correlation with clinical findings. Am J Trop Med Hyg 1999; 60:960-966. 